\title{
Extensions of Quasi-Free Derivations on the CAR algebra
}

\author{
By
}

Geoffrey L. PRICE*

\begin{abstract}
We classify all of the infinitesimal generator extensions of a particular quasi-free derivation $\delta_{A}$ on the CAR (canonical anticommutation relations) algebra, where $A$ is an unbounded symmetric operator on a Hilbert space having deficiency indices $(1,1)$. We show that each of these extensions generates a strongly continuous one-parameter group of Bogoliubov transformations of the CAR algebra.
\end{abstract}

\section{$\S 1$. Introduction}

An unbounded derivation $\delta$ on a $C^{*}$-algebra $\mathfrak{A}$ is said to be a $*$-derivation if its domain $D(\delta)$ is a dense *-subalgebra of $\mathfrak{A}$ and $\delta$ satisfies $\delta\left(x^{*}\right)=(\delta x)^{*}$ for all elements $x$ in its domain. Given a pair $\delta, \delta^{\prime}$ of $*$-derivations, we say that $\delta^{\prime}$ is an extension of $\delta$ if $D\left(\delta^{\prime}\right) \supseteqq D(\delta)$ and $\delta^{\prime} x=\delta x$ for all $x \in D(\delta)$. We shall say that $\delta$ is extendable if it admits an extension $\delta^{\prime}$ which is a generator of a $C^{*}$-dynamics, i. e., $\delta^{\prime}$ is the infinitesimal generator of a strongly continuous one-parameter group $\left\{\alpha_{t}: t \in \mathbb{R}\right\}$ of $*$-automorphisms of $\mathfrak{A}$.

P. E. T. Jørgensen has recently investigated the extendability of certain quasifree derivations $\delta_{A}$ on the CAR algebra $\mathscr{U}(\mathscr{H})(\mathscr{H}$ a separable Hilbert space) in terms of the deficiency indices $\left(n_{+}, n_{-}\right)$of the symmetric operator $A$ on $\mathscr{H}$. In [2] he showed that if an operator $A$ is maximal symmetric but not self-adjoint (i. e., $A$ has deficiency indices $(n, 0)$ or $(0, n)$ with $n>0)$ then $\delta_{A}$ is not extendable. It remains to be determined whether $\delta_{A}$ is extendable if $A$ has unequal indices $\left(n_{+}, n_{-}\right)$with $n_{+} \cdot n_{-} \neq 0$.

A related problem is the classification of all generator extensions of a quasifree derivation $\delta_{A}$, where $A$ has equal deficiency indices. Specifically, one would like to determine how such extensions are related to extensions of the symmetric operator $A$. In this paper we consider the particular example $A=i(d / d x)$ on $\mathscr{I}=L^{2}(0,1)$ with domain $D(A)=\{f \in A C[0,1]: f(0)=0=f(1)\} . \quad(A C[0,1]$ is the set of absolutely continuous functions on $[0,1]$.) $A$ has deficiency indices $(1,1)$. We show that any generator extension $\delta^{\prime}$ of $\delta_{A}$ gives rise to a one-parameter group $\left\{\alpha_{t}\right\}$ of Bogoliubov transformations, and that these extensions are in one-

Communicated by H. Araki, July 1, 1982.

* Department of Mathematics, I. U.P.U.I., Indianapolis, IN, U.S.A. 
to-one correspondence with a subset of the self-adjoint extensions of $A \oplus A$ on the Hilbert space $\mathscr{H} \oplus \overline{\mathcal{H}}$.

This work was completed while the author was a graduate student at the University of Pennsylvania. He wishes to thank his thesis supervisor, Professor Robert $T$. Powers, for acquainting him with this problem and for his numerous helpful observations. We are also grateful to P.E. T. Jørgensen for sending us a preprint of [2], and to $\mathrm{H}$. Araki for communicating to us a simpler proof of our Corollary 3.

\section{§2. Quasi-Free Derivations on the CAR Algebra}

Let $\mathscr{H}$ be a complex separable infinite-dimensional Hilbert space, with inner product $\langle$,$\rangle linear in the first component. We define \mathfrak{U}=\mathfrak{U}(\mathscr{H})$ to be the $C^{*}$ algebra of the canonical anti-commutation relations (CAR algebra). $\mathfrak{A}$ is generated by the range of the isometric linear mapping $f(\in \mathscr{H}) \mapsto a(f) \in \mathfrak{A}$, where the operators $\{a(f): f \in \mathscr{H}\}$ satisfy the relations

$$
\begin{gathered}
\left\{a(f)^{*}, a(g)\right\}_{+}=a(f)^{*} a(g)+a(g) a(f)^{*}=\langle g, f\rangle I, \\
\{a(f), a(g)\}_{+}=0 .
\end{gathered}
$$

The CAR algebra is a UHF-algebra of Glimm type $2^{\infty}$; in fact, if $\left\{f_{n}: n \in \boldsymbol{N}\right\}$ is an orthonormal basis for $\mathscr{H}$, define

$$
\begin{aligned}
U_{k} & =I-2 a\left(f_{k}\right) * a\left(f_{k}\right) \\
V_{0} & =I \\
V_{n} & =\prod_{k=1}^{n} U_{k} .
\end{aligned}
$$

Let $B_{n}$ be the $2 \times 2$ matrix algebra generated by $a\left(f_{n}\right) V_{n-1}, n \in N$ : then $B_{m}, B_{n}$ are commuting subalgebras for $m \neq n$, and we have $\mathfrak{A}=\bigotimes_{n \geqq 1}^{*} B_{n}$ (see $[6, \S 1]$ ).

Let $p \in \mathscr{U}$ be a polynomial in the operators $\left\{a(f)^{*}+a(g): f, g \in \mathscr{T}\right\}$ : then using the relations (1), if necessary, one may assume that $p$ is written as a linear combination of monomials of the form $a\left(g_{n}\right) * \cdots a\left(g_{1}\right) * a\left(h_{1}\right) \cdots a\left(h_{m}\right), g_{j}, h_{\imath} \in \mathscr{H}$, i. e., the starred operators are ordered to the left of the unstarred operators. We shall say that such a monomial (and the corresponding polynomial $p$ ) is in Wick order.

One may define an involution $\gamma$ on the operators $a(f)$ by $\gamma a(f)=-a(f)$. This involution extends to a $*$-automorphism (also denoted by $\gamma$ ) of the CAR algebra $\mathfrak{A}$, see [5]. We shall say that an element $x$ is even if $\gamma x=x$ and odd if $\gamma x=-x$. In particular, a Wick-ordered monomial $a\left(g_{n}\right) * \cdots a\left(g_{1}\right) * a\left(h_{1}\right) \cdots a\left(h_{m}\right)$ is even if $n+m$ is even, and odd if $n+m$ is odd. Any element $x$ can be decomposed as a sum $x=([\gamma x+x] / 2)+([x-\gamma x] / 2)$ of an even and an odd element.

Now suppose $A$ is an unbounded symmetric operator on $\mathscr{H}$, with dense domain $D(A)$. Letting $L=\left\{a(g)^{*}+a(f): g, f \in D(A)\right\}$, we define a linear opezator 
$\delta_{A}$ on $L$ by $\delta_{A}\left[a(g)^{*}+a(f)\right]=-i a(A g)^{*}+i a(A f)$. Extending the definition of $\delta_{A}$ to polynomials generated by the operators in $L$ by using the derivation identity $\delta(x y)=(\delta x) y+x \delta y$, one constructs a well-defined $*$-derivation $\delta_{A}$ with dense domain in $\mathfrak{A}$, the quasi-free derivation associated with $A$. The operator $\delta_{A}$ is closable (see [3, Corollary 2]); we shall also use the notation $\delta_{A}$ for the closure $\bar{\delta}_{A}$.

Finally, suppose that $U, V$ are linear and conjugate-linear partial isometries, respectively, which satisfy the consistency relations

$$
\begin{aligned}
& U^{*} U+V^{*} V=I=U\left(U^{*}\right)+V\left(V^{*}\right) ; \\
& U^{*} V+V^{*} U=0=U\left(V^{*}\right)+V\left(U^{*}\right) .
\end{aligned}
$$

The operators, $U, V$ induce a $*$-automorphism $\beta_{U, V}$ of the CAR algebra, the Bogoliubov transformation, defined by $\beta_{U, V} a(f)=a(U f)+a(V f)^{*}$. As an example, let $A$ be a symmetric operator on $\mathscr{H}$ admitting a self-adjoint extension $H$, and let $\left\{U_{t}: t \in \boldsymbol{R}\right\}$ be the strongly continuous one-parameter group of unitary operators on $\mathcal{H}$ generated by $H$, i. e., $U_{t}=e^{i t_{H}}$. For $t \in \mathbb{R}$ let $\beta_{t}$ be the Bogoliubov transformation $\beta_{U_{t}, 0}$. Then $\delta_{H}$ is the infinitesimal generator of the one-parameter group $\left\{\beta_{t}\right\}$ of $*$-automorphisms, so that $\delta_{A}$ is extendable.

\section{$\S 3 . \quad$ Extensions of a Quasi-Free Derivation}

In this section we restrict attention to the case where $\mathscr{H}=L^{2}(0,1)$, and $A$ is the symmetric operator $i(d / d x)$ on $\mathscr{A}$ with domain $D(A)=\{f \in A C[0,1]: f(0)=$ $0=f(1)\}$. (Here $A C[0,1]$ denotes the set of absolutely continuous functions on $[0,1]$.) Then it is well-known (see, e.g., $[7],[8]$ ) that $A$ is a closed symmetric operator with deficiency indices $(1,1)$, and deficiency subspaces $\mathscr{I}_{+}=\left\{c e^{x}: c \in C\right\}$, $\mathscr{F}_{-}=\left\{c e^{-x}: c \in \mathbb{C}\right\}$, where $\mathscr{F}_{ \pm}=[(i I \pm A) D(A)]^{\perp}$. Let $\delta_{A}$ be the (closed) quasi-free *-derivation associated with $A$. For the remainder of the discussion, let $\delta$ be an extension of $\delta_{A}$ generating a strongly continuous one-parameter group $\left\{\alpha_{t}: t \in \mathbb{R}\right\}$ of *-automorphisms of $\mathfrak{A}$.

In the following lemma we shall show that, for $t$ sufficiently small, $\alpha_{t}$ agrees with the Bogoliubov transformation of translation by $t$ on part of $\mathfrak{X}(\mathscr{H})$. First, we set down the following notation, to be used throughout this section.

Definition 1. For $t \in[0,1]$, let $U_{t}$ be the unitary translation operator defined on $f \in \mathscr{A}$ by $\left(U_{t} f\right)(x)=f(x-t)$, for $x \in[t, 1]$, and $\left(U_{t} f\right)(x)=f(x+1-t)$, for $x \in$ $[0, t]$. Let $\beta_{t}=\beta_{U_{t}, 0}$ be the corresponding Bogoliubov transformation on $\mathfrak{A}$.

Lemma 1. Preserve the notation above. Let $s \in[0,1)$ be fixed and suppose that $f \in \mathscr{H}$ is a function supported in $[0,1-s]$. Then for $0 \leqq t \leqq s, \alpha_{t} a(f)=\beta_{t} a(f)$.

Proof. By continuity it suffices to prove the lemma for $f \in D(A)$ satisfying the above conditions. For such $f$, note that $U_{t} f \in D(A)$, if $0 \leqq t \leqq s$, hence $\beta_{t} a(f)$ $\in D\left(\delta_{A}\right)$. Taking derivatives at $t=0$, we have $(d / d t)\left[\alpha_{t} a(f)\right]=\delta_{A} a(f)=-a\left(f^{\prime}\right)=$ $(d / d t)\left[\beta_{t} a(f)\right]$. Thus, defining $\gamma_{t}$ to be the automorphism $\gamma_{t}=\alpha_{-t} \circ \beta_{t}$, for $0 \leqq t \leqq s$, 
we have

$$
\begin{aligned}
\lim _{t \rightarrow 0} \frac{\gamma_{t} a(f)-a(f)}{t} & =\lim _{t \rightarrow 0} \frac{\alpha_{-t} a\left(U_{t} f\right)-a(f)}{t} \\
& =\lim _{t \rightarrow 0} \alpha_{-t}\left\{\frac{a\left(U_{t} f\right)-a(f)}{t}\right\}+\left\{\frac{\alpha_{-t} a(f)-a(f)}{t}\right\} \\
& =-\alpha_{0} a\left(f^{\prime}\right)+a\left(f^{\prime}\right)=0 .
\end{aligned}
$$

Similarly, one shows that for $0 \leqq r \leqq s, \gamma_{t+r} a(f)=\alpha_{-r}\left[\alpha_{-t} a\left(U_{t}\left(U_{r} f\right)\right)\right]$ has derivative 0 at $t=0$. Hence, for any $\phi \in \mathfrak{U}^{*}, \phi\left(\gamma_{t} a(f)\right)$ is differentiable with respect to $t$ for $0 \leqq t \leqq s$, and has constant derivative 0 . Thus $\phi\left(\gamma_{t} a(f)\right)=\phi\left(\gamma_{0} a(f)\right)=\phi(a(f))$, all $\phi \in \mathfrak{X}^{*}$, all $t \in[0, s)$, so that $a(f)=\gamma_{t} a(f)=a_{-t} \beta_{t} a(f)$, and therefore $\alpha_{t} a(f)=\beta_{t} a(f)$ $=a\left(U_{t} f\right)$ for $t \in[0, s)$.

Remark. Let $[a, b]$ be a subinterval of $[0,1]$, and let $\chi_{[a, b]}$ be the characteristic function on $[a, b]$. The lemma above shows that for $t$ sufficiently small, i. e., $0 \leqq t \leqq 1-b, \alpha_{t} a\left(\chi_{[a, b]}\right)=a\left(\chi_{[a+t, b+t]}\right)$. The following lemmas are directed towards determining the action of $\alpha_{t}$ on the one-particle operators $a\left(\chi_{[a, b]}\right)$, for more general $t$.

Let $P$ be an orthogonal projection on $\mathscr{K}$, and let $\left\{f_{i}\right\}_{i=1}^{n}$ (with $n$ possibly infinite) be an orthonormal basis for the range of $P$. The set $\left\{a\left(f_{i}\right)\right\}_{i=1}^{n}$ generates a $C^{*}$-subalgebra of $\mathfrak{A}$, which we shall denote by $\mathfrak{A}(P)$. (For $P=0$, set $\mathfrak{A}(0)=$ $\{c I: c \in C\}$.) Note that if $n$ is finite, $\mathfrak{X}(P)$ is a $2^{n} \times 2^{n}$ matrix subalgebra of $\mathfrak{A}$.

Definition 2. Let $\mathfrak{B}$ be a $C^{*}$-subalgebra of $\mathfrak{A}$. Then $\mathfrak{B}^{c}$, the relative commutant of $\mathfrak{B}$ with respect to $\mathfrak{A}$, is given by $\mathfrak{B}^{\mathfrak{c}}=\{x \in \mathfrak{A}: x y=y x$, all $y \in \mathfrak{B}\}$.

One may show, by using the anti-commutation relations (1), that if $x$ is an even polynomial in $\mathfrak{X}(I-P)$, and $y$ any polynomial in $\mathfrak{X}(P)$, then $x y=y x$. By continuity, the same holds for any $y \in \mathfrak{U}(P)$, so that $x \in \mathfrak{X}(P)^{c}$. Now suppose $x$ is any even element of $\mathfrak{X}(I-P)$. Then $x$ is a limit of polynomials $\left\{q_{n}: n \in \mathbb{N}\right\}$ in $\mathfrak{X}(I-P)$, hence $x=(\gamma x+x) / 2=\lim _{n \rightarrow \infty}\left(\gamma q_{n}+q_{n}\right) / 2$ is a limit of even polynomials in $\mathfrak{X}(I-P)$, so that $x \in \mathfrak{A}(P)^{c}$, by continuity. In the following two lemmas we establish a partial converse. The statements and proofs of these lemmas are due to $\mathrm{H}$. Araki, and simplify our original argument.

Lemma 2. If $\operatorname{dim} P=\infty$, and $x \in \mathfrak{U}(P)^{c}$, then $x$ is even.

Proof. Let $\left\{f_{n}: n \in N\right\}$ be an orthonormal system for $P \mathscr{H}$, so that wk- $\lim _{n \rightarrow \infty} f_{n}$ $=0$. Then $u_{n}=a\left(f_{n}\right)+a\left(f_{n}\right)^{*} \in \mathfrak{X}(P)$ is a self-adjoint unitary element, and using (1) a straightforward argument shows that for any polynomial $y$ (of creation and annihilation operators), $\gamma y=\lim _{n \rightarrow \infty} u_{n} y\left(u_{n}\right)^{*}$. By continuity, the same holds for any element $y \in \mathfrak{A}$. In particular, for $x \in \mathfrak{A}(P)^{c} ; \gamma x=\lim _{n \rightarrow \infty} u_{n} x\left(u_{n}\right)^{*}=x$, so that $x$ is even. 
Lemma 3. If $\operatorname{dim} P=\infty$ and $x \in \mathfrak{A}(P)^{c}$, or if $x \in \mathfrak{U}(P)^{c}$ and $\gamma(x)=x$, then $x \in \mathfrak{X}(I-P)$.

Proof. Let $\varepsilon>0$. Since $\mathfrak{A}$ is the uniform closure of polynomials in $\mathfrak{A}(P)$ and $\mathfrak{Y}(I-P)$, we may choose a finite-rank subprojection $P_{\varepsilon}$ of $P$ and a polynomial $x_{\varepsilon}$ in the subalgebra generated by $\mathfrak{A}\left(P_{\varepsilon}\right)$ and $\mathfrak{A}(I-P)$ such that $\left\|x-x_{\varepsilon}\right\|$ $<\varepsilon$. Since $\gamma x=x$ (by assumption or by Lemma 2), we may assume that $x_{\varepsilon}$ is also even. Furthermore, applying (1) we may assume that $x_{\varepsilon}$ has the form $\sum_{i=1}^{r} y_{i} z_{i}$, where the $y_{i} \in \mathfrak{U}\left(P_{\varepsilon}\right)$ (respectively, the $z_{i} \in \mathfrak{U}(I-P)$ ) are monomials.

Since $\mathfrak{A}\left(P_{\varepsilon}\right)$ is a matrix algebra (hence a Type I factor) there exists a unitary $u \in \mathfrak{U}\left(P_{\varepsilon}\right)$ implementing the automorphism $\gamma$, i. e., $\gamma(y)=u y(u)^{*}, y \in \mathfrak{X}\left(P_{\varepsilon}\right)$. Setting $y=u$, we have $\gamma(u)=u$, and therefore (by the remarks preceding Lemma 2) $u \in \mathfrak{A}(I-P)^{c}$. Set $x_{\varepsilon}^{\prime}=\left(x_{\varepsilon}+u x_{\varepsilon}(u)^{*}\right) / 2$, then clearly $\left\|x-x_{\varepsilon}^{\prime}\right\|<\varepsilon, x_{\varepsilon}^{\prime}$ is even, and

$$
x_{\varepsilon}^{\prime}=\sum_{i=1}^{r}\left[\left(y_{i}+u y_{\imath}(u)^{*}\right) / 2\right] z_{i} .
$$

We may thus write $x_{\varepsilon}^{\prime}=\sum_{i=1}^{r^{\prime}} y_{\imath}^{\prime} z_{i}^{\prime}$, where $y_{i}^{\prime} \in \mathfrak{A}\left(P_{\varepsilon}\right)$ is even, and $z_{\imath}^{\prime} \in \mathfrak{A}(I-P)$. Since $x_{\varepsilon}^{\prime}$ is even, we may assume that the $z_{i}^{\prime}$ are also even.

Now set $x_{\varepsilon}^{\prime \prime}=\int v x_{\varepsilon}^{\prime}\left(v^{*}\right) d v$, where $d v$ is normalized Haar measure on the group of unitaries of $\mathfrak{A}\left(P_{\varepsilon}\right)$. Then since $x \in \mathfrak{A}(P)^{c}$,

$$
\left\|x-x_{\varepsilon}^{\prime \prime}\right\|=\left\|\int v\left(x-x_{\varepsilon}^{\prime}\right) v^{*} d v\right\| \leqq \int\left\|v\left(x-x_{\varepsilon}^{\prime}\right) v^{*}\right\| d v=\int\left\|x-x_{\varepsilon}^{\prime}\right\| d v<\varepsilon .
$$

Moreover, since $z_{\imath}^{\prime} \in \mathfrak{A}(I-P)$ is even, $v z_{\imath}^{\prime}=z_{\imath}^{\prime} v$, all $v \in \mathfrak{A}\left(P_{\varepsilon}\right)$, and therefore

$$
\begin{aligned}
x_{\varepsilon}^{\prime \prime} & =\sum_{i=1}^{r^{\prime}}\left[\int v y_{i}^{\prime} z_{\imath}^{\prime}\left(v^{*}\right) d v\right] \\
& =\sum_{i=1}^{r^{\prime}}\left[\int v y_{\imath}^{\prime}\left(v^{*}\right) d v\right] z_{i}^{\prime} \\
& =\sum_{i=1}^{r^{\prime}} y_{\imath}^{\prime \prime} z_{\imath}^{\prime},
\end{aligned}
$$

where $y_{\imath}^{\prime \prime}=\int v y_{\imath}^{\prime}\left(v^{*}\right) d v$. By invariance of Haar measure, one easily checks that $y_{i}^{\prime \prime} \in \mathfrak{A}\left(P_{\varepsilon}\right)$ commutes with the unitary group of the factor $\mathfrak{A}\left(P_{\varepsilon}\right)$. hence with all of $\mathfrak{A}\left(P_{\varepsilon}\right)$, and therefore the elements $y_{i}^{\prime \prime}$ are scalar operators. Hence $x_{\varepsilon}^{\prime \prime}$ is an even element of $\mathfrak{A}(I-P)$. Since $\varepsilon$ is arbitrary, the same properties hold for $x$, by continuity, and we are done.

Corollary. Let $P$ be an infinite-dimensional projection on $\mathscr{H}$. Then $\mathfrak{A}(P)^{c}$ consists of the even elements of $\mathfrak{A}(I-P)$.

Proof. Combine Lemmas 2 and 3. 
For fixed $n \in N$, and $1 \leqq k \leqq n$, let $E_{k}$ be the projection on $\mathscr{H}$ given by $E_{k} f$ $=\chi_{[(k-1) / n, k / n]} f$. Then the subalgebra $\mathfrak{X}\left(I-E_{n}\right)$ of $\mathfrak{U}$ satisfies $\alpha_{(1 / n)}\left(\mathfrak{U}\left(I-E_{n}\right)\right)$ $=\mathfrak{A}\left(I-E_{1}\right)$, by Lemma 1 , since $\alpha_{(1 / n)} a(f)=a(U(1 / n) f)$, all $f \in\left(I-E_{n}\right) \mathscr{H}$. Therefore, denoting by $\mathfrak{B}_{e}$ the even elements of a subalgebra $\mathfrak{B}$ of $\mathfrak{A}$, we have, applying Corollary 3, $\alpha_{(1 / n)}\left[\mathfrak{A}\left(E_{n}\right)_{e}\right]=\alpha_{(1 / n)}\left[\mathfrak{A}\left(I-E_{n}\right)^{c}\right]=\left[\alpha_{(1 / n)} \mathfrak{A}\left(I-E_{n}\right)\right]^{c}=\mathfrak{A}\left(I-E_{1}\right)^{c}$ $=\mathfrak{A}\left(E_{1}\right)_{e}$. Moreover, suppose $y \in \mathfrak{A}\left(E_{n}\right)$ is odd: then $y$ (resp., $\alpha_{(1 / n)} y$ ) commutes with the even and anti-commutes with the odd elements of $\mathfrak{A}\left(I-E_{n}\right)$ (respectively, of $\left.\alpha_{(1 / n)} \mathfrak{A}\left(I-E_{n}\right)=\mathfrak{A}\left(I-E_{1}\right)\right)$. Let $g \in E_{1} \mathscr{K}$ be a unit vector, then (letting $a(g)^{\#}$ denote either $a(g)$ or $\left.a(g)^{*}\right) a(g)^{\#}$ commutes with $\mathfrak{Y}\left(I-E_{1}\right)_{e}$ and anti-com-

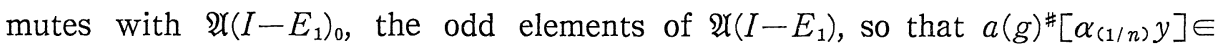
$\mathfrak{U}\left(I-E_{1}\right)^{c}=\mathfrak{U}\left(E_{1}\right)_{e} . \quad$ Therefore, $\alpha_{(1 / n)} y=a(g) *\left[a(g) \alpha_{(1 / n)} y\right]+a(g)\left[a(g) * \alpha_{(1 / n)} y\right] \in$ $\mathfrak{X}\left(E_{1}\right)$, so that $\alpha_{(1 / n)}\left[\mathfrak{X}\left(E_{n}\right)_{0}\right] \cong \mathfrak{X}\left(E_{1}\right)_{0}$. A similar argument shows that $\alpha_{(-1 / n)}\left[\mathfrak{A}\left(E_{1}\right)_{0}\right] \subseteq \mathfrak{U}\left(E_{n}\right)_{0}$, hence (since $\left.\alpha_{(-1 / n)} \circ \alpha_{(1 / n)}=i d\right) \alpha_{(1 / n)}$ maps $\mathfrak{A}\left(E_{n}\right)_{0}$ onto $\mathfrak{U}\left(E_{1}\right)_{0}$. Therefore we have the

Proposition 4. Let $\delta$ be an extension of $\delta_{A}$ generating a strongly continuous one-parameter group $\left\{\alpha_{t}: t \in \boldsymbol{R}\right\}$ of automorphisms of $\mathfrak{A}$. Then for fixed $n \in \mathbb{N}$, and projections $E_{k}(1 \leqq k \leqq n)$ defined as above, $\alpha_{(1 / n)} \mathfrak{A}\left(E_{n}\right)=\mathfrak{X}\left(E_{1}\right)$.

Let $\chi$ be the characteristic function $\chi_{[0,1]}$. As an application of the preceding result we shall explicitly determine $\alpha_{1} a(\chi)$. Using this we shall then show (in Theorem 7) that $\left\{\alpha_{t}\right\}$ is a one-parameter group of Bogoliubov transformations uniquely determined by $\alpha_{1} a(\chi)$.

The proof of the following lemma is motivated by the techniques in the proof of [4, Lemma 1].

Lemma 5. Let $\left\{\alpha_{t}\right\}, \chi$ be as above. Then $\alpha_{1} a(\chi)$ is a sum of one particle operators, i.e., $\alpha_{1} a(\chi)=a\left(h_{1}\right)+a\left(h_{2}\right)^{*}$, some $h_{1}, h_{2} \in \mathscr{H}$.

Proof. Let $y=\alpha_{1} a(\chi)$, let $n \in N$ be fixed, and for $1 \leqq k \leqq n$, denote by $\chi_{k}$ the characteristic function $\chi_{[(k-1) / n, k / n]}$. Then $y=\sum_{k=1}^{n} \alpha_{1} a\left(\chi_{k}\right)$. For $k<n$ we have, applying Lemma 1, $\alpha_{1} a\left(\chi_{k}\right)=\alpha_{(k / n)}\left[\alpha_{(n-k) / n} a\left(\chi_{k}\right)\right]=\alpha_{(k / n)}\left[\beta_{(n-k) / n} a\left(\chi_{k}\right)\right]=\alpha_{(k / n)}$. $\left[a\left(U(n-k / n) \chi_{k}\right)\right]=\alpha_{(k / n)} a\left(\chi_{n}\right)$, hence for all $k \leqq n, \alpha_{1} a\left(\chi_{k}\right)=\alpha_{(k-1) / n}\left[\alpha_{(1 / n)} a\left(\chi_{n}\right)\right]$. By Proposition 4, $\alpha_{(1 / n)} a\left(\chi_{n}\right)$ is an odd element of $\mathfrak{X}\left(E_{1}\right)$, since $a\left(\chi_{n}\right)$ is an odd element of $\mathfrak{A}\left(E_{n}\right)$. Then by another application of Lemma 1, we have $\alpha_{(k-1) / n} \mathfrak{A}\left(E_{1}\right)$ $=\beta_{(k-1) / n} \mathfrak{U}\left(E_{1}\right)=\mathfrak{U}\left(E_{k}\right)$, so that $\alpha_{1} a\left(\chi_{k}\right)=\alpha_{(k-1) / n}\left[\alpha_{(1 / n)} a\left(\chi_{n}\right)\right]$ is an odd element of $\mathfrak{X}\left(E_{k}\right)$. Let $y_{k}=\alpha_{1}\left(\chi_{k}\right)$ : then $y=\sum_{k=1}^{n} y_{k}$, where $y_{k} \in \mathfrak{X}\left(E_{k}\right)$ is odd, and $\left\|y_{k}\right\|=$ $\left\|\alpha_{1} a\left(\chi_{k}\right)\right\|=\left\|a\left(\chi_{k}\right)\right\|=\left\|\chi_{k}\right\|=(1 / \sqrt{n})$.

We shall now show that for all $f, g \in \mathscr{H},\left[a(f)^{\#},\left\{a(g)^{\#}, y\right\}_{+}\right]_{-}=0$. To show this it suffices to assume that $f$ and $g$ are characteristic functions of $[0,1]$, since linear combinations of these are dense in $L^{2}(0,1)$. By Lemma $A$ of the appendix (see also [4, Lemma 3]) it follows that $y$ is the sum of one-particle operators. For $1 \leqq k \leqq n$, let $g_{k}=E_{k} g, f_{k}=E_{k} f$. Then the elements $a\left(g_{k}\right)^{\#}, a\left(f_{k}\right) \#$ are odd 
elements of $\mathfrak{U}\left(E_{k}\right)$, and since $y_{l} \in \mathfrak{U}\left(E_{l}\right)$ is also odd, we have, for distinct $k$ and $l,\left\{a\left(g_{k}\right)^{\#}, y_{l}\right\}_{+}=a\left(g_{k}\right)^{\#} y_{l}+y_{l} a\left(g_{k}\right)^{\#}=0$. Therefore $\left\{a(g)^{\#}, y\right\}_{+}=\sum_{k=1}^{n} \sum_{l=1}^{n}\left\{a\left(g_{k}\right)^{\#}, y_{l}\right\}_{+}$ $=\sum_{k=1}^{n}\left\{a\left(g_{k}\right)^{\#}, y_{k}\right\}_{+}$. Now $\left\{a\left(g_{k}\right)^{\#}, y_{k}\right\}_{+}$is an even element of $\mathfrak{A}\left(E_{k}\right)$, hence it commutes with $a\left(f_{i}\right)^{\#}$, for $i \neq k$. Thus

$$
\begin{aligned}
{\left[a(f)^{\#},\left\{a(g)^{\#}, y\right\}_{+}\right]_{-} } & =\sum_{i=1}^{n} \sum_{k=1}^{n}\left[a\left(f_{i}\right)^{\#},\left\{a\left(g_{k}\right)^{\#}, y_{k}\right\}_{+}\right]_{-} \\
& =\sum_{k=1}^{n}\left[a\left(f_{k}\right)^{\#},\left\{a\left(g_{k}\right)^{\#}, y_{k}\right\}_{+}\right]_{-} .
\end{aligned}
$$

Since $f$ and $g$ are characteristic functions, $\left\|a\left(f_{k}\right)\right\|=\left\|f_{k}\right\| \leqq\left\|\chi_{k}\right\|=(1 / \sqrt{n})$, and similarly for $g_{k}$. Thus

$$
\begin{aligned}
\left\|\left[a(f)^{\#},\left\{a(g)^{\#}, y\right\}_{+}\right]_{-}\right\| & =\left\|\sum_{k=1}^{n}\left[a\left(f_{k}\right)^{\#},\left\{a\left(g_{k}\right)^{\#}, y_{k}\right\}_{+}\right]-\right\| \\
& \leqq 4 \cdot \sum_{k=1}^{n}\left\|a\left(f_{k}\right)\right\| \cdot\left\|a\left(g_{k}\right)\right\| \cdot\left\|y_{k}\right\| \\
& \leqq 4 n(1 / \sqrt{n})^{3}=4 / \sqrt{n} .
\end{aligned}
$$

Since this is true for all $n \in N,\left[a(f)^{\#},\left\{a(g)^{\#}, y\right\}_{+}\right]_{-}=0$, and the lemma is proved.

Lemma 6. There exists $\theta \in[0,2 \pi)$ such that either a) $\alpha_{1} a(\chi)=a\left(e^{i \theta} \chi\right)$ or b) $\alpha_{1} a(\chi)=a\left(e^{i \theta} \chi\right)^{*}$.

Proof. Fix $n \in N$, and suppose $y=\alpha_{1} a(\chi)=a\left(h_{1}\right)+a\left(h_{2}\right)^{*}, h_{1}, h_{2} \in \mathscr{A}$. By the proof of the preceding lemma, $y=\sum_{k=1}^{n} y_{k}$, where $y_{k}=\alpha_{(k / n)} a\left(\chi_{n}\right)=$ $\beta_{(k-1) / n}\left[\alpha_{(1 / n)} a\left(\chi_{n}\right)\right] \in \mathfrak{U}\left(E_{k}\right) . \quad$ It follows that $y_{1}=a\left(\chi_{1} h_{1}\right)+a\left(\chi_{1} h_{2}\right)^{*}, y_{k}=$ $a\left(U((k-1) / n) \chi_{1} h_{1}\right)+a\left(U((k-1) / n) \chi_{1} h_{2}\right)^{*}, 1 \leqq k \leqq n$, and so for all $n$,

$$
h_{i}=\sum_{k=1}^{n} U\left(\frac{k-1}{n}\right)\left(\chi_{[0,1 / n]} \cdot h_{i}\right), \quad i=1,2 .
$$

By a straightforward argument, (4) implies that $h_{1}, h_{2}$ are constant functions. Thus $\alpha_{1} a(\chi)=a\left(c_{1} \chi\right)+a\left(c_{2} \chi\right)^{*}, \quad c_{1}, c_{2} \in C$. Using (1), $0=\alpha_{1}\left[a(\chi)^{2}\right]=\left[\alpha_{1} a(\chi)\right]^{2}=$ $\left\langle c_{1} \chi, c_{2} \chi\right\rangle=c_{1} \bar{c}_{2}$, so $c_{1}=0$ or $c_{2}=0$. If $c_{2}=0,\left|c_{1}\right|=1$, since $1=\|a(\chi)\|=\left\|\alpha_{1} a(\chi)\right\|=$ $\left\|a\left(c_{1} \chi\right)\right\|=\left\|c_{1} \chi\right\|=\left|c_{1}\right|$. Similarly, if $c_{1}=0$, then $\left|c_{2}\right|=1$, and we are done.

Theorem 7. Let $\delta$ be a generator extension of $\delta_{A}$ on the CAR algebra $\mathfrak{A}$, with the corresponding strongly continuous one-parameter group $\left\{\alpha_{t}: t \in \mathbb{R}\right\}$ of *-automorphisms of $\mathfrak{X}$. Then for each $t \in \boldsymbol{R}, \alpha_{t}$ is a Bogoliubov transformation; for any $f \in \mathscr{H}$ and $0 \leqq t \leqq 1$, there is a $\theta \in[0,2 \pi)$ such that $\alpha_{t} a(f)$ must have one of the following forms:

a) $\alpha_{t} a(f)=a\left(\chi_{[t, 1]} U(t) f\right)+a\left(e^{i \theta} \chi_{[0, t]} U(t) f\right)$

b) $\alpha_{t} a(f)=a\left(\chi_{[t, 1]} U(t) f\right)+a\left(e^{i \theta} \chi_{[0, t]} \overline{U(t) f)^{*}}\right.$

$(\bar{g}$ denotes the conjugate function of $g \in \mathscr{K})$. 
Proof. The composition and inverses of Bogoliubov transformations are Bogoliubov, see [5]. Hence if the automorphisms $\alpha_{t}, 0 \leqq t \leqq 1$, are Bogoliubov, it follows that the entire group $\left\{\alpha_{t}: t \in R\right\}$ consists of Bogoliubov transformations. On the other hand, if $\alpha_{t}$ satisfies a) or $\mathrm{b}$ ), for $0 \leqq t \leqq 1$, then using (3) it is straightforward to verify that $\alpha_{t}$ is Bogoliubov.

So suppose $t \in[0,1]$. Using strong continuity of $\left\{\alpha_{t}\right\}$ it suffices to show that $\alpha_{t}$ has the required properties a) or b) for $t \in \boldsymbol{Q}$ (the rational numbers). As a further simplification, we need only check the result for $f$ of the form $f=c \chi_{[a, b]}$, where $a, b \in \boldsymbol{Q} \cap[0,1]$, since finite sums of such functions are dense in $\mathscr{A}$. Let $n$ be a common denominator for $a, b, t$. Retaining the notation of the previous lemma, and applying Lemma 1 and Proposition 4, $\alpha_{t} a\left(c \chi_{[a, b]}\right)$ is determined by $\alpha_{(1 / n)} a\left(\chi_{n}\right)$. Suppose $\alpha_{1} a(\chi)=a\left(e^{i \theta} \chi\right)$ : then $y_{1}=\alpha_{(1 / n)} a\left(\chi_{n}\right)=a\left(e^{i \theta} \chi_{1}\right)$, and $\alpha_{t} a\left(c \chi_{[a, b]}\right)$ is easily shown to satisfy a). If $\alpha_{1} a(\chi)=a\left(e^{i \theta} \chi\right)^{*}$, then $\alpha_{(1 / n)} a\left(\chi_{n}\right)=a\left(e^{i \theta} \chi_{1}\right)^{*}$, and $\alpha_{t}$ has form $\left.\mathrm{b}\right)$. This completes the theorem.

Remark. For completeness we sketch without proof the correspondence between generator extensions $\delta$ of $\delta_{A}$, whose associated one-parameter groups are given in Theorem 7, and extensions of the symmetric operator $A$. We omit the proofs, since they are straightforward but rather tedious. For this note we assume familiarity with von Neumann's results on the classification of self-adjoint extensions of a symmetric operator (see [8] for a treatment of this).

It is well known (see, for example, [7], [8]) that a one-to-one correspondence exists between numbers $\theta \in[0,2 \pi)$ and self-adjoint extensions $T_{\theta}$ of $A$, where $T_{\theta}$ is the operator $i(d / d x)$ on its domain $D\left(T_{\theta}\right)=\left\{f \in A C[0,1]: f(0)=e^{i \theta} f(1)\right\}$. For fixed $\theta \in[0,2 \pi)$, let $\delta_{T_{\theta}} \supseteqq \delta_{A}$ be the quasi-free derivation associated with $T_{\theta}$. Then, since $T_{\theta}$ is self-adjoint, $\delta_{T_{\theta}}$ is a generator extension of $\delta_{A}$, and $\delta_{T_{\theta}}$ generates a one-parameter group of Bogoliubov transformations $\left\{\alpha_{t}: t \in \mathbb{R}\right\}$ satisfying $\alpha_{t} a(f)=a\left(e^{i t T \theta} f\right)$, all $f \in \mathscr{H}$. It is straightforward to check that $e^{i T} \theta=e^{i \theta} \chi$, so that $\alpha_{1} a(\chi)=a\left(e^{i \theta} \chi\right)$. Thus the extensions $\delta \supseteqq \delta_{A}$ whose automorphism groups are of the form in part a) of Theorem 7 coincide with the quasi-free derivations $\delta_{T_{\theta}}$, for $\theta \in[0,2 \pi)$.

We sketch the situation for part b) of the theorem. Let $\overline{\mathscr{H}}$ be the Hilbert space conjugate to $\mathscr{K}$, and $H$ the operator $H=A \oplus A$ on the Hilbert space $\mathscr{H} \oplus \overline{\mathcal{H}}$ with domain $D(H)=D(A) \oplus \overline{D(A)}$. Then for $\{f, \bar{g}\} \in D(H), H(\{f, \bar{g}\})=\{A f, A \bar{g}\}$. $H$ is symmetric and has deficiency subspaces $\mathscr{T}_{+}=\left\{\left\{c e_{+}, \overline{d e_{+}}\right\}: c, d \in \mathbb{C}\right\}, \mathscr{T}_{-}=$ $\left\{\left\{c e_{-}, \overline{d e_{-}}\right\}: c, d \in C\right\} \quad$ where $e_{+}=\exp (x) /\|\exp (x)\| ; e_{-}=\exp (-x) /\|\exp (-x)\|$; and $\mathscr{F}_{ \pm}=[(i(I \oplus I) \pm H) D(H)]^{\perp}$. For fixed $\theta \in[0,2 \pi)$, let $H_{\theta}$ be the operator $i(d / d x) \oplus i(d / d x)$ on $\mathscr{T} \oplus \overline{\mathcal{H}}$ with domain $D\left(H_{\theta}\right)$ the linear manifold spanned by $D(H),\left\{e_{+}, \overline{e^{i \theta} e_{-}}\right\}$, and $\left\{e^{i \theta} e_{-}, \bar{e}_{+}\right\}$. Then $H_{\theta}$ is a self-adjoint operator extending $H$ : this follows, for example, by noting that the Cayley transform $(H-i I)(H+i I)^{-1}$ (resp., $\left.(H+i I)(H-i I)^{-1}\right)$ maps $\mathscr{I}_{+}$isometrically onto $\mathscr{F}_{-}$(respectively, $\mathscr{F}_{-}$isometrically onto $\mathscr{I}_{+}$). 
If $\left\{\gamma_{t}: t \in \boldsymbol{R}\right\}$ is the one-parameter group of unitary operators generated by $H_{\theta}$, and $\{f, \overline{0}\} \in \mathscr{H} \oplus \overline{\mathcal{H}}$, then for $t \in[0,1]$, one may verify that $\gamma_{t}(\{f, \overline{0}\})=$ $\left\{\chi_{[t, 1]} U(t) f, e^{-i \theta} \overline{\chi_{[0, t]} U(t) f}\right\}$. Next we may identify $\mathscr{H} \oplus \overline{\mathcal{H}}$ with the one-particle operators $\left\{a(f)+a(g)^{*}: f, g \in \mathscr{H}\right\}$ via the continuous linear embedding mapping $\{f, \bar{g}\}$ to $\left[a(f)+a(g)^{*}\right]$. Under this identification the unitary group $\left\{\gamma_{t}: t \in \mathbb{R}\right\}$ corresponds to the Bogoliubov transformations in part b), and we are done.

The following conjecture (of Powers) is a plausible extension of the result of Jørgensen mentioned in the introduction, and of Theorem 7.

Conjecture. Let $S$ be a symmetric operator on a Hilbert space $\mathscr{H}$, and let $\delta_{S}$ be the corresponding quasi-free derivation on $\mathfrak{A}(\mathscr{H})$. Then $\delta_{S}$ is extendable if and only if $S$ has equal deficiency indices. If $\delta \supseteqq \delta_{S}$ is a generator of a strongly continuous one-parameter group $\left\{\alpha_{t}: t \in \mathbb{R}\right\}$ of $*$-automorphisms on $\mathfrak{U}(\mathscr{H})$, then for each $t, \alpha_{t}$ is a Bogoliubov transformation.

It appears likely, however, that the proof of the general case will require some techniques beyond those used here. For our particular example, essential use was made of the fact that for small $t, \alpha_{t}$ acts like a Bogoliubov transformation on operators $a(f)$, where $f \in \mathscr{H}$ is a function supported away from the endpoints. In order to proceed with the general case along the lines of this paper, one would need an analogue of this result: i. e., if $\delta \supseteqq \delta_{S}$ is a generator extension of a quasi-free derivation $\delta_{S}$, with $\left\{\alpha_{t}: t \in \mathbb{R}\right\}$ its corresponding one-parameter group, one would need to show that for small $t, \alpha_{t}$ acts like (or "approximately like") a Bogoliubov transformation on a "large" subalgebra of $\mathfrak{A}$. Such a result seems difficult to obtain.

\section{Appendix}

Lemma A. Let $\mathscr{H}$ be a separable Hilbert space, $\mathfrak{A}(\mathscr{H})$ the CAR algebra over $\mathscr{H}$, and suppose $y \in \mathfrak{X}(\mathscr{H})$ satisfies $\left[a(f)^{\#},\left\{a(g)^{\#}, y\right\}_{+}\right]_{-}=0$, all $f, g \in \mathscr{H}$, where $a(f)^{\#}$ denotes either $a(f)$ or $a(f)^{*}$. Then $y$ is a sum of one-particle operators, i.e., there exist $h_{1}, h_{2} \in \mathscr{A}$ such that $y=a\left(h_{1}\right)+a\left(h_{2}\right)^{*}$.

Proof. Fix $g \in \mathscr{H}$, then for all $f \in \mathscr{H},\left[a(f)^{\#},\left\{a(g)^{\#}, y\right\}_{+}\right]_{-}=0$, so that $\left\{a(g)^{\#}, y\right\}_{+}$commutes with all of the $a(f)^{\#}$. Therefore, $\left\{a(g)^{\#}, y\right\}_{+}$lies in the center of $\mathfrak{A}(\mathscr{H})$, which, by Corollary 3 , consists only of scalar multiples of the identity. Thus $\left\{a(g)^{\#}, y\right\}_{+}=\phi^{\#}(g) I$, where $\psi$ is a linear (respectively, $\psi^{*}$ is a conjugate-linear) functional on $\mathscr{H}$. For $g \in \mathscr{H},\left|\phi^{\#}(g)\right|=\left\|\left\{a(g)^{\#}, y\right\}_{+}\right\| \leqq 2\|y\|$. $\left\|a(g)^{\#}\right\|=2\|y\|\|g\|$, so that both $\psi$ and $\psi^{*}$ are continuous. By the Riesz Lemma there exist vectors $h_{1}, h_{2} \in \mathscr{H}$ such that, for all $g \in \mathscr{H}, \phi(g)=\left\langle g, h_{1}\right\rangle$ (resp., $\phi^{*}(g)$ $=\left\langle h_{2}, g\right\rangle$ ). Letting $z$ be the operator $z=y-\left[a\left(h_{1}\right)+a\left(h_{2}\right)^{*}\right]$, a straightforward calculation shows that $\left\{a(g)^{\#}, z\right\}_{+}=0$, for all $g \in \mathcal{H}$. We show that $z=0$. Let $I \neq P \neq 0$ be an orthogonal projection, and suppose that $h=P h$ is a unit vector. 
Then for any $g \in(I-P) \mathcal{H}, a(g)^{\#} a(h)^{\#} z=-a(h)^{\#} a(g)^{\#} z=a(h)^{\#} z a(g)^{\#}$, so that $a(h)^{\#} z \in \mathfrak{X}(I-P)^{c}$, and therefore (Corollary 3) $a(h)^{\#} z \in \mathfrak{X}(P)$. Since $a(h)^{\#} \in \mathfrak{U}(P)$, we have $z=a(h)[a(h) * z]+a(h) *[a(h) z] \in \mathfrak{X}(P)$. A similar argument shows $z \in$ $\mathfrak{U}(I-P)$, and thus $z \in \mathfrak{X}(P) \cap \mathfrak{U}(I-P)=\{c I: c \in C\}$. But $\left\{a(g)^{\#}, z\right\}_{+}=0$ for all $g \in \mathscr{H}$ then implies that $z=0$, and we are done.

\section{References}

[1] Hugenholtz, N.M. and Kadison, R. V., Automorphisms and quasi-free states of the CAR-algebra, Comm. Math. Phys., 43 (1975), 181-197.

[2] J $\phi$ rgensen, P.E.T., Extensions of unbounded *-derivations in UHF $C^{*}$-algebras, J. Funct. Anal., 45 (1982), 341-356.

[3] Kishimoto, A., Dissipations and derivations, Comm. Math. Phys., 47 (1976), 2532.

[4] Powers, R. T., Absence of interaction as a consequence of good ultraviolet behavior in the case of a local Fermi field., Comm. Math. Phys., 4 (1967), 145-156.

[5] - Representations of the canonical anticommutation relations, Thesis, Princeton University (1967).

[6] Powers, R.T. and St $\phi$ rmer, E., Free states of the canonical anticommutation relations, Comm. Math. Phys., 16 (1970), 1-33.

[7] Reed, M. and Simon, B., Methods of modern mathematical physics, 2, Academic Press, New York, 1975.

[8] Riesz, F. and Sz.-Nagy, B., Functional analysis, Frederick Ungar, New York, 1955. 